Casos Clínicos

Arch. Esp. Urol., 60, 2 (185-186), 2007

\section{MANEJO DE UN PACIENTE CON ESTRANGULACIÓN DE PREPUCIO POR CREMALLERA.}

Jeff R. Cortés González, Jorge Arratia Maqueo, Lauro Gómez Guerra y Rodolfo Jaime Dávila.

Hospital Universitario "Dr. José E. González UANL".

Monterrey, Nuevo León. México.

Resumen.- OBJETIVO: Demostrar que la estrangulación del prepucio por la cremallera requiere atención inmediata por especialistas.

MÉTODOS: Presentación de un caso de un paciente de 8 años sin antecedentes de importancia que acude tras presentar estrangulación del pene con la cremallera del pantalón.

RESULTADOS: Posterior a múltiples intentos de retirar la cremallera bajo sedación, el paciente decide acudir al servicio de urología. El paciente es sometido a intervención quirúrgica evolucionando satisfactoriamente.

CONCLUSIÓN: La eventual atención oportuna, la individualización de cada caso en particular, la experiencia del médico tratante dictara la conducta a seguir en cada

Jeff R. Cortés González

Servicio de Urología

Hospital Universitario "José E. González" UANL

Av, Madero y Gonzalitos s/n

Col. Mitras Centro.

64460 Monterrey. NL. (México).

ircor77@yahoo.com, jeff.cortes@urologiahu.org

Trabajo recibido: 12 de abril 2006 paciente. Se debe hacer consciencia en el personal médico de las salas de urgencias tanto pediátricas como de adultos de que es un problema que requiere atención inmediata y se debe evitar realizar tratamientos o abordajes empíricos y/o heróicos.

Palabras clave: Estrangulación de pene. Trauma genital. Cremallera.

Summary.- OBJECTIVE: To demonstrate that strangulation of the prepuce needs immediate specialist care.

METHODS: Presentation of a 8-years-old boy case with an entrapped foreskin in his pant's zipper.

RESULTS: After multiple attempts to remove the zipper under sedation, the case was referred to our service. The patient was surgically treated and his evolution was good.

CONCLUSIONS: Immediate care, individualization of each case, as well as the experience of the treating physician will dictate the approach to follow in each patient. Medical personnel in adult as well as pediatric emergency departments must be aware that it is a problem that requires immediate care and avoid doing empirical and/or heroic treatments.

Keywords: Penile strangulation. Genital Trauma. Zipper injury.

\section{INTRODUCCIÓN}

Uno de los problemas con los que se encuentra el médico en las salas de urgencias es el trauma genital, particularmente cuando hay estrangulación del prepucio por cremallera (1-4). Muchos abordajes se han descrito para tratar este problema como soluciones aceitosas (3), algunos instrumentos especializados para romper la parte media de la cremallera, la circuncisión, etc. Los cuales varían dependiendo de la presentación del cuadro clínico, y el tiempo de evolución. En este artículo presentamos el caso de un niño de 8 años con estrangulación de prepucio por cremallera.

\section{PRESENTACIÓN DEL CASO Y MANEJO}

\section{CASO}

Paciente masculino de 8 años de edad sin antecedentes médicos de importancia, el cual posterior a ir efectuar la micción, se sube de manera abrupta la cremallera del pantalón y se produce estrangulación del prepucio. Cinco horas después avisa a sus padres del accidente. Al momento de evidenciar lesión es trasladado inmediatamente al servicio de urgencias de nuestro 

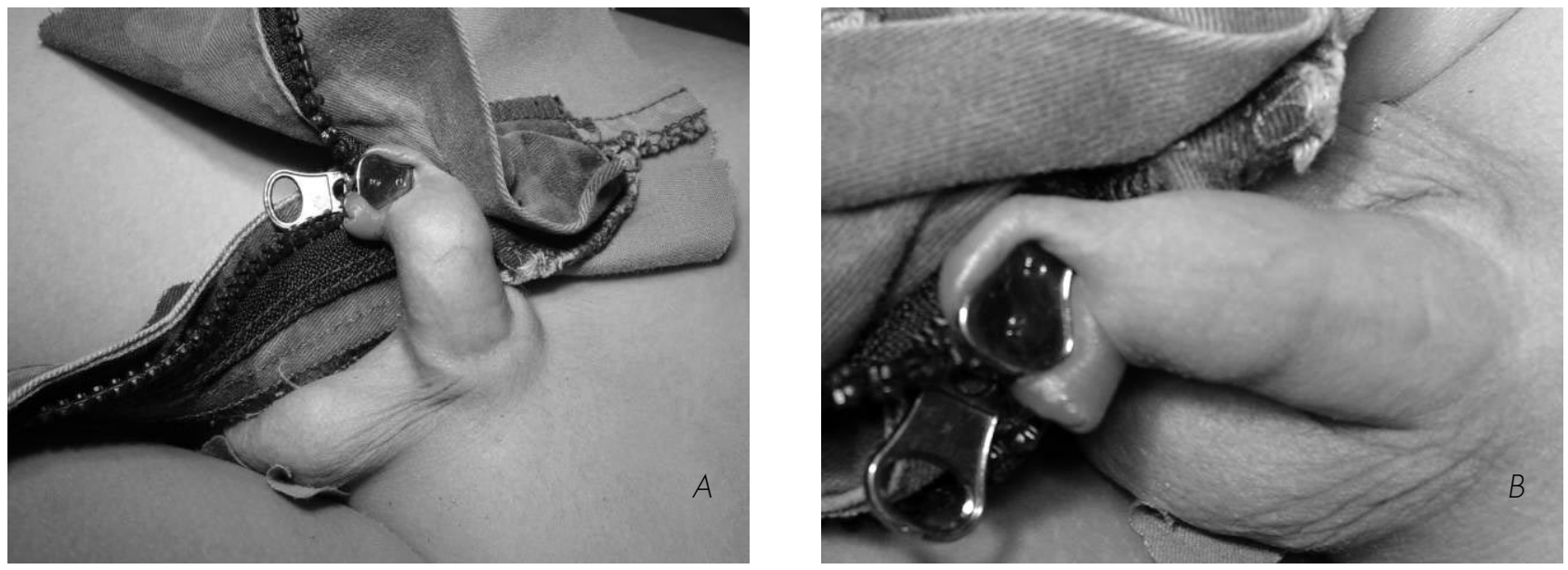

FIGURA IAI. Se aprecia pene con prepucio estrangulado con pantalón recortado.

B). Acercamiento de fotografía previa donde se aprecia ligero edema de prepucio y la estrangulación franca del mismo.

hospital. Debido a dolor importante y para facilitar el traslado, la madre decide cortar pantalón y dejar solamente la cremallera (Figura 1 A). Posterior a múltiples intentos de retirar la cremallera bajo sedación, el paciente es trasladado al servicio de urología. Al momento de la valoración encontramos al paciente con el pene edematizado, estrangulado entre dientes de la cremallera (Figura 1 B). El paciente se encontraba ansioso y poco cooperador. Se decidió la intervención quirúrgica. Se le realizó la circuncisión inmediatamente posterior a la valoración obteniendo un resultado postoperatorio satisfactorio. (Figura 2).

\section{DISCUSIÓN}

La estrangulación de prepucio por cremallera es un problema común con el que se encuentra el médico en la

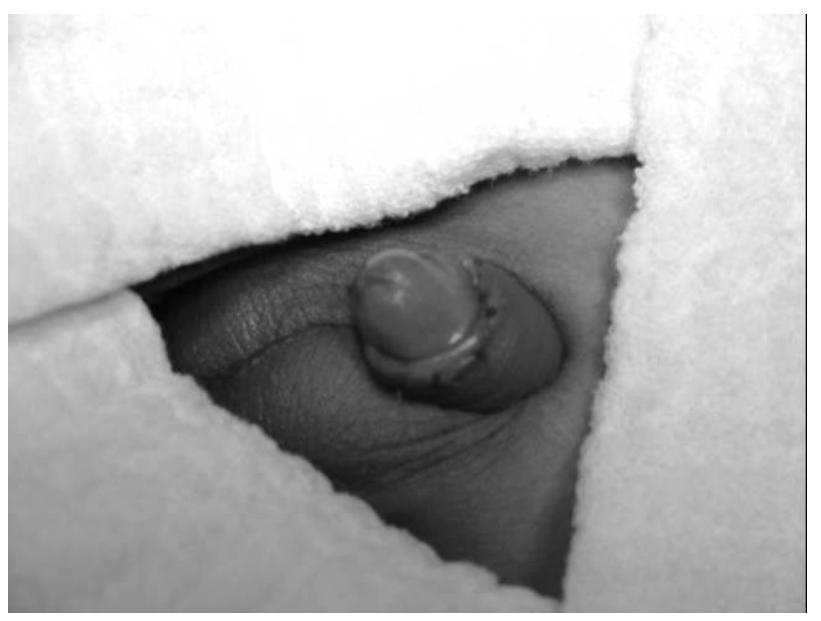

FIGURA 2. Aspecto postoperatorio de pene tras circuncisión. sala de urgencias (1). A pesar de que en la literatura se han descrito diferentes manejos para la resolución de este problema (2-4). La eventual atención oportuna, la individualización de cada caso en particular, así como la experiencia del médico tratante es lo que dictara la conducta a seguir en cada paciente. Se debe concienciar al personal médico de las salas de urgencias tanto pediátricas como de adultos de que es un problema que requiere atención inmediata, que tratar de realizar tratamientos o abordajes empíricos y/o heróicos, puede diferir el tratamiento apropiado y repercutir en la eventual evolución del paciente.

\section{BIBLIOGRAFÍA y LECTURAS RECOMENDADÁS (*lectura de interés $y^{* *}$ lectura fundamental)}

*1. NOLAN, J.F.; STILLWELL, T.J.; SANDS, J.P. Jr.: "Acute management of the zipper entrapped penis". J. Emer. Med.; 8:305. 1990.

**2. STRAIT, R.T.: "Pediatric emergency care. A novel method for removal of penile zipper entrapment". Pediatr Emerg Care.; Vol. 15, pp. 412. 1999.

*3. KANEGAYE, J.T.; SCHONFELD, N.: "Penile zipper entrapment: a simple and less threatening approach using mineral oil". Pediatr Emerg Care.; Vol. 9, pp. 90. 1993.

4. OOSTERLINCK, W.: "Unbloody management of penile zipper injury". Eur Urol.; Vol. 7, pp. 365. 1981. 